\title{
Implementing California Senate Bill 493 in an Outpatient Pharmacy Within an Integrated Health System: Evaluation of the Operational and Clinical Effect of Pharmacist-Ordered Laboratory Tests
}

\author{
Hector M. Delgado, PharmD; Nicole P. Kleinman, PharmD; \\ Abir F. Makarem, PharmD; and Courtney Nguyen, BA
}

\begin{abstract}
BACKGROUND: Under California Senate Bill 493, pharmacists can order patient laboratory tests (labs). Currently, it is unknown if this service affects patient outcomes or pharmacy operations. Does lab ordering by pharmacists improve access to care, improve quality outcomes, and/or affect pharmacy operational functions?

PROGRAM DESCRIPTION: A 13-month pilot study was conducted at 2 Kaiser Permanente (KP) outpatient pharmacies where pharmacists provided extended adherence consultations and ordered hemoglobin A1c (HbA1c) labs for patients nonadherent to their oral diabetic medications with an $\mathrm{HbA1c} \geq 8 \%$ or missing annual labs. Clinical outcomes of the pilot study were compared with a similar patient population at KP who concurrently received lab orders from their primary providers, defined here as the "usual care model."

OBSERVATION: Of the $793 \mathrm{HbA1c}$ lab orders, 87 (11.0\%) were generated by a pharmacist, and $706(89.0 \%)$ were generated by the usual care group. Forty-three (49.4\%) patients in the pharmacist group completed their labs compared with 279 (39.5\%) patients in the usual care group $(P=0.10)$. A significantly greater proportion of patients in the pharmacist group achieved an $\mathrm{HbA1c}<8 \%$ within the follow-up period of $30-180$ days $(34.9 \%)$, compared with the usual care group $(12.2 \%, P<0.01)$. Of the patients who completed labs during the evaluation period, $38(43.7 \%)$ patients in the pharmacist group and $111(15.7 \%)$ patients in the usual care group had prelaboratory values $\geq 8 \%$ within the previous 12 months. The average pre- $\mathrm{HbA1c}$ value was $9.47 \%$ in the pharmacist group, and the average post-HbA1c value was $8.68 \%(P<0.01)$. For the usual care group, the average pre- $\mathrm{HbA} 1 \mathrm{c}$ value was $9.70 \%$, and the average post-HbA1c value was $9.43 \%(P=0.06)$. When comparing the difference in $\mathrm{HbA1c}$ reduction between the 2 groups, there was a larger decrease in $\mathrm{HbA1c}$ in the pharmacist group, but this difference was not significantly different $(P=0.06)$. The pilot study added an average of 5 minutes per patient encounter to the pharmacy workflow but did not affect overall patient wait times for receiving outpatient prescriptions.
\end{abstract}

IMPLICATIONS: Laboratory ordering by pharmacists in the outpatient setting improved access to care, improved quality outcomes, and did not adversely affect pharmacy operations.

J Manag Care Spec Pharm. 2020;26(3):320-24

Copyright $\odot 2020$, Academy of Managed Care Pharmacy. All rights reserved.

\section{What is already known about this subject}

California Senate Bill 493, passed in 2013, allows pharmacists to order and interpret laboratory tests (labs), furnish birth control and smoking cessation products, and administer a wide variety of vaccines without a physician protocol for patients aged 3 years and over.

Based on the language in California Assembly Bill 1114, it is uncertain how pharmacists will be reimbursed for providing lab ordering services.

The lack of access to medical records in the community pharmacy setting may be a barrier to implementation of various services recognized by Senate Bill 493.

\section{What this study adds}

Pharmacists are able to positively affect clinical outcomes for patients through lab ordering in the outpatient pharmacy setting. Lab ordering by pharmacists in the outpatient pharmacy could be operationally sustainable, but potential barriers, such as volume of lab orders and a lack of reimbursement, may limit this service in traditional independent and chain pharmacies.

$\mathrm{P}$ harmacists in California have had an expanded scope of practice since the passing of California Senate Bill (SB) 493 in 2013. ${ }^{1}$ Through this expanded scope of practice, pharmacists are able to order and interpret labs, furnish birth control and smoking cessation products, and administer a wide variety of vaccines without a physician protocol for patients aged over 3 years. However, only a minority of pharmacists participate in these services. A study by Gomez (2017) identified that only $11 \%$ of community-based, retail pharmacies offered pharmacist-prescribed hormonal contraception. ${ }^{2}$ Rodriguez et al. (2016) looked at barriers to implementing pharmacist-furnished hormonal contraception and identified a lack of pharmacy staff, concerns about liability, and the need for additional training as some of the most common factors that impeded pharmacist participation. ${ }^{3}$

While there are several studies investigating pharmacist perceptions or economic evaluations of SB 493 implementation, ${ }^{4-6}$ a search of the literature rendered limited information 
on laboratory test (lab) ordering services and their relationship with outpatient operations or clinical patient outcomes. This is likely because of the difficulty that independent and community pharmacies have in accessing patient medical records, which makes this type of study challenging. Moreover, a bill does not currently exist in California that would provide reimbursement for lab ordering services provided by pharmacists, potentially hindering them from offering the service altogether.

At Kaiser Permanente (KP), pharmacists in the outpatient setting have access to the electronic medical record (EMR), KP HealthConnect, to order and review patient labs. However, even within an integrated system such as KP, where improving patient medication use outcomes are integrated into the workflow, incremental resources are needed to accommodate for pharmacists' time in providing these services. While allowing pharmacists to work at the top of their license would increase access to care, reduce care gaps, and improve quality outcomes, ${ }^{7,8}$ these outcomes have yet to be studied with regards to lab ordering in the outpatient setting. Our pilot program aimed to implement outpatient pharmacist lab ordering coupled with face-to-face consultations for patients nonadherent to their oral diabetes medications with a hemoglobin Alc ( $\mathrm{HbAlc}) \geq 8 \%$ or a missing annual HbAlc. Our objectives were to report on the operational effect of the program and assess its effect on clinical patient outcomes as measured by HbAlc goals.

\section{Program Description}

The pilot study was conducted at 2 sites from October 1, 2016 through October 31, 2017-KP Los Angeles Medical Center 4900 Sunset Pharmacy and KP Baldwin Park 24-Hour Pharmacy. Ten pharmacists engaged in this pilot and were required to complete a 60 -minute online training for diabetes competency and a 60-minute live online session on lab ordering and results follow-up in KP HealthConnect. Patients included in this study belonged to a KP outpatient pharmacy clinical service (OPCS) program that targets patients with diabetes who have suboptimal rates of oral antidiabetic medication adherence, as measured by a medication possession ratio (MPR) of $<80 \%$ and an $\mathrm{HbAlc}$ lab result $\geq 8 \%$, or missing annual HbAlc. ${ }^{9}$ MPR is defined as the percentage of the total days supply for all fills of a given drug in a particular time period divided by the number of days in the time period. ${ }^{10}$

\section{Traditional Workflow}

When a patient in the OPCS program was identified during prescription processing for any medication in the pharmacy, a care management summary sheet was generated, displaying the most recent labs for HbAlc, low-density lipoprotein cholesterol, and a list of the 20 most recent prescription fills within the previous 12 months. In addition, the summary sheet displayed the respective MPRs and days supply remaining for the patient's chronic medications. At the time that patients picked up any requested medication, the pharmacist provided an extended face-to-face consultation to identify and address barriers to adherence, along with an explanation of what an HbAlc result signified and its respective goals for patients with diabetes. In this "usual care model," the primary care provider then ordered a monitoring or screening HbAlc lab for patients who agreed to restart their nonadherent medications as prescribed. To assess outcomes, patients had to receive OPCS consultations and complete HbAlc lab orders placed by their providers within 30 to 180 days after their consultations.

\section{Pilot Study Workflow}

With the new workflow designed to implement the expanded scope of practice given to pharmacists under SB 493, pharmacists in the pilot program ordered the monitoring lab at the time of the face-to-face extended consultation for patients who agreed to restart an oral diabetes medication rather than request an order from the provider. This bypassed the need for a potential office visit or provider contact to order such labs. Four weeks after patients restarted their medications, they could visit any KP laboratory for the lab test. The results were then routed electronically through KP HealthConnect to the pharmacists, where they could electronically connect with patients' providers to determine the need for follow-up and potential medication dosage change. Patients were followed from day 30 to day 180 after the lab orders were placed. Patients who did not complete labs during the observation period were excluded in the analysis for HbAlc outcomes.

\section{Pharmacist Perspectives}

To assess operational effects and job satisfaction of pharmacists involved in this pilot program, a survey consisting of seven 5-level Likert scale questions was conducted at the pilot sites. The pharmacy manager at each pharmacy collected the surveys from the participating pharmacists and submitted the results to the pilot program's coinvestigators. Also, an interview was conducted with one of the pharmacists who participated in the pilot program.

\section{Goals and Objectives}

The primary goal was to determine if OPCS lab ordering by pharmacists improved access to care, as measured by the number of completed labs ordered by a pharmacist. In addition, this pilot program aimed to look at how this expanded role of outpatient pharmacists could improve quality outcomes, as measured by HbAlc. This study also looked at the pilot program's effect on outpatient pharmacy operational functions and the satisfaction of pharmacists participating in the program.

\section{Clinical Review}

The coinvestigators performed a clinical review process through a protocol approved by the KP Southern California 


\begin{tabular}{|c|c|c|c|}
\hline TABLE 1 & $\begin{array}{l}\text { Demographic } \\
\text { Included in the } \\
\text { (October 1, } 20\end{array}$ & $\begin{array}{l}\text { Data for Patient } \\
\text { Pilot Period } \\
\text { 16-October 31, }\end{array}$ & $017)$ \\
\hline Variable & $\begin{array}{c}\text { Pharmacist Group } \\
\mathbf{n}=87, \% \text { (n) }\end{array}$ & $\begin{array}{c}\text { Usual Care Group } \\
\mathbf{n}=706, \%(\mathrm{n})\end{array}$ & $P$ Value ${ }^{\mathrm{a}}$ \\
\hline Sex, male & $55.2(48)$ & $53.7(379)$ & 0.88 \\
\hline Age, mean [SD] & $61[12.4]$ & $58[12.6]$ & 0.04 \\
\hline \multicolumn{4}{|l|}{ Race/ethnicity } \\
\hline Asian & $5.7(5)$ & $12.6(89)$ & 0.09 \\
\hline Black & $2.3(2)$ & $4.7(33)$ & 0.46 \\
\hline Hispanic/Latino & $66.7(58)$ & $62.7(443)$ & 0.55 \\
\hline White & $14.9(13)$ & $12.0(85)$ & 0.55 \\
\hline Native American & $2.3(2)$ & $2.4(17)$ & 0.95 \\
\hline Other & $4.6(4)$ & $4.1(29)$ & 0.83 \\
\hline Declined to state & $3.5(3)$ & $1.4(10)$ & 0.34 \\
\hline
\end{tabular}

Institutional Review Board. The process involved retrospective chart reviews for the following patient-specific information: age, sex, self-reported ethnicity and race, date of lab order and completion, laboratory result, and prelaboratory date and value within the previous 12 months, if applicable. An HbAlc value $<8 \%$ was the target outcome for all patients, regardless of age or comorbidities. ${ }^{11}$ Lab ordering by pharmacists was the only independent variable tested in this study.

\section{Statistical Analysis}

Continuous variables were compared between groups using an unpaired 2-tailed t-test and within groups using a paired 2-tailed t-test. Categorical variables were analyzed for statistically significant differences between groups using the chisquare test with Yates correction. Statistical significance was defined using $P \leq 0.05$.

\section{Observations}

During the study period (October 1, 2016-October 31, 2017), 793 patients were included. Table 1 illustrates demographic data for patients who had laboratory tests ordered by either pharmacists or the usual care group. The pharmacist group was composed of 55\% males, compared with 54\% in the usual care group. Race and ethnicity were self-reported by patients, with Hispanic/Latino being the largest proportion of patients at $67 \%$ and $63 \%$ for the pharmacist and usual care groups, respectively. The differences between the groups were not found to be significant ( $P>0.05$ for all reported races). The average age was higher in the pharmacist group at 61 years compared with 58 years for the usual care group. This finding was statistically significant $(P=0.04)$.

\section{Quality and Clinical Outcomes}

Of the $793 \mathrm{HbAlc}$ lab orders, 87 (11.0\%) were generated by a pharmacist, whereas 706 (89.0\%) lab orders were generated by the usual care group (Table 2). Forty-three (49.4\%) of 87 patients in the pharmacist group completed their laboratory draws, compared with 279 (39.5\%) of 706 patients in the usual care group $(P=0.10)$. A significantly greater proportion of patients in the pharmacist group achieved an HbAlc $<8 \%$ (34.9\%), compared with the usual care group $(12.2 \%, P<0.01)$. Of the patients who completed labs during the evaluation period, 38 (43.7\%) patients in the pharmacist group and 111 (15.7\%) patients in the usual care group had prelaboratory values $\geq 8 \%$ within the previous 12 months $(P<0.01)$.

The pharmacist group had an average pre-HbAlc value of $9.47 \%$ and an average post-HbAlc value of $8.68 \%$, with an average decrease of 0.79 percentage points, which was found to be statistically significant $(P<0.01)$. For the usual care group, the average pre-HbAlc was $9.70 \%$ and the average post-HbAlc was $9.43 \%$, with an average decrease of 0.27 percentage points. This difference was not statistically significant $(P=0.06)$. When comparing the difference in HbAlc reduction between the 2 groups, there was a larger decrease in the pharmacist group, but this difference was not significantly different from the usual care group $(P=0.06)$. The average time lapse between the date the lab order was placed and the post-HbAlc lab draw was 82 days for the pharmacist group and 79 days for the usual care group. This difference between the 2 groups was not statistically significant $(P=0.43)$.

\section{Pharmacist Perspective}

Review of the pharmacist survey showed that pharmacists strongly agreed that engagement with HbAlc lab ordering is valuable and will improve quality of care, patient safety, clinical outcomes, and access to needed care. A major barrier to patient participation within the pilot program identified in the survey was nonadherence to completing lab draws due to lab fees. From the perspective of the pharmacists, the survey revealed the preference for a KP HealthConnect lab ordering training in a formal, live learning environment.

The pharmacist who was interviewed discussed how electronic lab ordering was complicated and how in-person training may have helped facilitate this process. The pharmacist was pleased with the pilot program stating, "We are able to review clinical aspects of the patient in the chart. The only thing is will time allow it, when we have other competing priorities in our outpatient pharmacy. It is sometimes difficult, when it is really busy, to give your undivided attention."

\section{Operational Effect}

Participating in the pilot program required 1 hour of additional workflow training per pharmacist. According to the survey results, the OPCS extended consultation added an average of 


\section{Implementing California Senate Bill 493 in an Outpatient Pharmacy Within an Integrated Health System: Evaluation of the Operational and Clinical Effect of Pharmacist-Ordered Laboratory Tests}

\begin{tabular}{|c|c|c|c|}
\hline Variable & Pharmacist Group & Usual Care Group & $P$ Value ${ }^{\mathrm{a}}$ \\
\hline Labs ordered, ${ }^{\mathrm{b}} \mathrm{n}$ & 87 & 706 & \\
\hline Patients who completed laboratory draws within the evaluation period, \% (n) & $49.4 \quad(43)$ & $39.5 \quad(279)$ & 0.10 \\
\hline Lab results with $\mathrm{HbAlc}<8 \%, \%(\mathrm{n})$ & 34.9 & 12.2 & $<0.01$ \\
\hline Mean number of days from lab order placement to lab completion, days [SD] & [32] & [39] & 0.43 \\
\hline $\begin{array}{l}\text { Patients with prelaboratory HbAlc values } \geq 8 \% \text { within the previous } \\
12 \text { months and completed labs during the evaluation period, \% (n) }\end{array}$ & (38) & (111) & $<0.01$ \\
\hline Change in $\mathrm{HbAlc}$ from pre to post, ${ }^{\mathrm{c}}$ [SD] (\% change) & $9.47[1.52]$ to 8.68 [1.35] (8.3) & & $<0.01$ \\
\hline Change in HbAlc from pre to post, ${ }^{\mathrm{c}}$ [SD] (\% change) & & $9.70[1.51]$ to $9.43[1.57](2.8)$ & 0.06 \\
\hline Mean change in HbAlc from pre to post,c,d [SD] & [1.26] & $\begin{array}{ll}-0.27 & {[1.50]} \\
\end{array}$ & 0.06 \\
\hline \multicolumn{4}{|c|}{$\begin{array}{l}\text { aUnpaired two-tailed } t \text {-test for means data between groups, paired two-tailed } t \text {-test for means data within groups, and chi-square test with Yates correction for categorical } \\
\text { data. } \\
\text { bFor the pharmacist group, the number of labs ordered were based on the total successful consults resulting in HbAlc lab orders. } \\
\text { cThe variances calculated included patients who had prelaboratory HbAlc values } \geq 8 \% \text { within the previous } 12 \text { months and lab orders placed and completed during the pilot } \\
\text { program (pharmacist group, } n=38 \text {, and usual care group, } n=111 \text { ). } \\
\text { dMean change was determined by calculating the variance of each individual pair of HbAlc values for each group. } \\
\text { HbAlc=hemoglobin Alc; OPCS=Outpatient Pharmacy Clinical Services; SD=standard deviation. }\end{array}$} \\
\hline
\end{tabular}

5 minutes to the prescription workflow from start to finish. The resulting HbAlc labs were routed via KP HealthConnect to a message pool, where pharmacists in that outpatient pharmacy could access while onsite. The 5-minute OPCS extended consultation did not affect the pharmacies' productivity metrics, as measured by the overall wait time for patients to request and pay for their completed prescriptions.

\section{Implications}

Age was the only demographic that was significantly different between the pharmacist and usual care groups. A larger percentage of patients in the pharmacist group completed their lab orders relative to the usual care group. Moreover, the pharmacist group had a significant decrease in HbAlc, which was not seen in the usual care group. When comparing the difference in $\mathrm{HbAlc}$ reduction between the 2 groups, the drop in $\mathrm{HbAlc}$ was greater in the pharmacist group, but this difference did not reach significance. Our findings demonstrate the effect pharmacists have not only on potentially increasing access to care, as measured by completed lab orders, but also on improving clinical outcomes, as measured by a decrease in HbAlc. Our search of the literature did not find any studies addressing these outcomes.

The relatively higher average age of patients in the pharmacist group may demonstrate the benefit pharmacists have on providing clinical care to older adults. Older adults can have multiple chronic conditions that require several medications and can benefit from a pharmacist's clinical interventions. With the implementation of Medicare Part D in 2006, pharmacists have been able to provide medication therapy management (MTM) services to these patients. MTM does not involve the ordering of labs, but studies have shown that MTM services, including restarting nonadherent medications, can improve clinical outcomes. Pinto et al. (2014) demonstrated that MTM services after 1 year decreased patient HbAlc by 0.27 percentage points. ${ }^{12}$ The pilot program resulted in an HbAlc reduction within the pharmacist group that was almost 3 times the magnitude relative to the Pinto et al. study.

Interestingly, the survey and the interview revealed that pharmacists prefer hands-on training with regards to lab ordering. Since SB 493 came to fruition in 2014, ${ }^{1}$ ordering and interpreting laboratory tests was likely not included in the pharmacy education curriculum. Moreover, because lab ordering in the outpatient setting has only been implemented in a pilot setting, new KP employees are not trained on lab ordering as part of the onboarding process.

Outpatient pharmacists in the community have had challenges implementing lab ordering into their practice for several reasons. Pharmacies would require access to patient medical records. For KP, the integrated medical system facilitates lab ordering and the access of results through the synced EMR. Moreover, O'Connor et al. (2012) identified barriers to implementing pharmacogenetic lab ordering in the outpatient pharmacy setting that included the lack of insurances recognizing pharmacists as health care providers, which would prevent reimbursement and even ordering of such labs. ${ }^{13}$ With the passing of California Assembly Bill 1114 in 2016, pharmacist services including immunizations, smoking cessation counseling, and furnishing of travel medication, hormonal contraception, and nicotine replacement therapy will be reimbursed through Medi-Cal by July 1, 2021. ${ }^{14}$ While this bill provides an outlet for pharmacists to be reimbursed for their services in the outpatient setting, it is uncertain, based on the language of the bill, how lab ordering services will be reimbursed. 
The 5-minute encounter time did not affect patient wait times in the pharmacy, but it did add a workflow step for the pharmacists. The lack of a payment model for lab ordering by pharmacists creates a barrier for implementation within outpatient pharmacies. While KP does not rely on reimbursement for individual pharmacist-provided services, if lab ordering were to increase in volume and scaled up to include other lab tests, incremental resources to meet the changing workflow needs would be necessary.

Pharmacist intervention through outpatient lab ordering showed a positive effect on improved lab draw adherence and $\mathrm{HbAlc}$ reduction. However, these outcomes may be influenced by several confounding factors, including patient age, sex, race, and comorbidities. Furthermore, while the pharmacist intervention significantly reduced $\mathrm{HbAlc}$ values, the average post-HbAlc was still $\geq 8 \%$. Additional pharmacist services may be necessary to further reduce HbAlc values in order to achieve clinical targets. Limitations of the pilot study include the smaller size of the pharmacist group compared with the usual care group and the large proportion of patients in both groups who did not complete ordered labs. Future randomized studies will be necessary to address differences in group size and possible confounding variables, as well as reasons for low completion rates of ordered labs.

In conclusion, engaging pharmacists in lab ordering in the outpatient pharmacy setting improves access to care, enhances quality outcomes, and does not adversely affect pharmacy operations. Pharmacists who participated in this pilot program had a positive experience and would benefit from the opportunity to provide lab ordering services to patients.

\section{Authors}

HECTOR M. DELGADO, PharmD; NICOLE P. KLEINMAN, PharmD; ABIR F. MAKAREM, PharmD; and COURTNEY NGUYEN, BA, Kaiser Permanente, Los Angeles, California.

AUTHOR CORRESPONDENCE: Hector M. Delgado, PharmD, Kaiser Permanente, 1515 N. Vermont Ave., Ste. 237, Los Angeles, CA 90027. Tel.: 323.783.5684; E-mail: Hector.M.Delgado@kp.org.

\section{DISCLOSURES}

No outside funding supported this project. The authors have nothing to disclose.

\section{REFERENCES}

1. California Legislative Information. SB-493 Pharmacy practice. October 2013. Available at: https://leginfo.legislature.ca.gov/faces/billTextClient. xhtml?bill_id=201320140SB493. Accessed January 31, 2020

2. Gomez AM. Availability of pharmacist-prescribed contraception in California, 2017. JAMA. 2017;318(22):2253-54.

3. Rodriguez MI, McConnell KJ, Swartz J, Edelman AB. Pharmacist prescription of hormonal contraception in Oregon: baseline knowledge and interest in provision. J Am Pharm Assoc. 2016;56(5):521-26.

4. Raebal MA, Chester EA, Newsom EE, et al. Randomized trial to improve laboratory safety monitoring of ongoing drug therapy in ambulatory patients. Pharmacotherapy. 2006;26(5):619-26.

5. Vu K, Rafie S, Grindlay K, Gutierrez H, Grossman, D. Pharmacist intentions to prescribe hormonal contraception following new legislative authority in California. J Pharm Pract. 2019;32(1):54-61.

6. Fixen DR, Linnebur SA, Parnes BL, Vejar MV, Vande Griend JP. Development and economic evaluation of a pharmacist-provided chronic care management service in an ambulatory care geriatrics clinic. Am J Health Syst Pharm. 2018;75(22):1805-11.

7. Hess KM, Dai CW, Garner B, Law AV. Measuring outcomes of a pharmacist-run travel health clinic located in an independent community pharmacy. J Am Pharm Assoc. 2010;50(2):174-80

8. Murray MD, Young J, Hoke S, et al. Pharmacist intervention to improve medication adherence in heart failure: a randomized trial. Ann Intern Med. 2008; 146(10):714-25.

9. Spence MM, Makarem AF, Reyes SL, et al. Evaluation of an outpatient pharmacy clinical services program on adherence and clinical outcomes among patients with diabetes and/or coronary artery disease. J Manag Care Spec Pharm. 2014;20(10):1036-45. Available at: https://www.jmcp.org/ doi/10.18553/jmcp.2014.20.10.1036

10. Crowe M. Do you know the difference between these adherence measures? Pharmacy Times. July 5, 2015. Available at: https://www.pharmacytimes.com/contributor/michael-crowe-pharmd-mba-csp-fmpa/2015/07/ do-you-know-the-difference-between-these-adherence-measures. Accessed January 31, 2020.

11. American Diabetes Association. 6. Glycemic targets: standards of medical care in diabetes-2018. Diabetes Care. 2018;41(Suppl 1):S55-S64.

12. Pinto SL, Kumar J, Partha G, Bechtol RA. Pharmacist-provided medication therapy management (MTM) program impacts outcomes for employees with diabetes. Popul Health Manag. 2014;17(1):21-27.

13. O'Connor SK, Ferreri SP, Michaels NM, et al. Making pharmacogenetic testing a reality in community pharmacy. J Am Pharm Assoc. 2012; 52(6):e259-65.

14. California Legislative Information. AB-1114 Medi-Cal: pharmacist services. September 2016. Available at: https://leginfo.legislature.ca.gov/faces/ billTextClient.xhtml?bill_id=201520160AB1114. Accessed January 31, 2020. 